\title{
Identification of a Novel Fusarium Head Blight Resistance Quantitative Trait Locus on Chromosome 7A in Tetraploid Wheat
}

\author{
S. Kumar, R.W. Stack, T. L. Friesen, and J. D. Faris
}

First author: Department of Plant Sciences, North Dakota State University, Fargo 58105; second author: Department of Plant Pathology, North Dakota State University, Fargo 58105; and third and fourth authors: U.S. Department of Agriculture-Agricultural Research Service, Cereal Crops Research Unit, Northern Crop Science Laboratory, Fargo, ND 58105. Accepted for publication 29 November 2006.

\begin{abstract}
Kumar, S., Stack, R. W., Friesen, T. L., and Faris, J. D. 2007. Identification of a novel Fusarium head blight resistance quantitative trait locus on chromosome 7A in tetraploid wheat. Phytopathology 97:592-597.

Fusarium head blight (FHB) caused by Fusarium graminearum is one of the most destructive diseases of durum (Triticum turgidum sp. durum) and common wheat (T. aestivum). Promising sources of FHB resistance have been identified among common (hexaploid) wheats, but the same is not true for durum (tetraploid) wheats. A previous study indicated that chromosome 7A from T. turgidum sp. dicoccoides accession PI478742 contributed significant levels of resistance to FHB. The objectives of this research were to develop a genetic linkage map of chromosome $7 \mathrm{~A}$ in a population of 118 recombinant inbred lines derived from a cross between

dicoccoides PI478742 chromosome 7A substitution line [LDN-DIC 7A(742)], and identify a putative FHB resistance quantitative trait locus (QTL) on chromosome 7A derived from LDN-DIC 7A(742). The population was evaluated for type II FHB resistance in three greenhouse environments. Interval regression analysis indicated that a single QTL designated $Q$ fhs. $f c u-7 A L$ explained $19 \%$ of the phenotypic variation and spanned an interval of $39.6 \mathrm{cM}$. Comparisons between the genetic map and a previously constructed physical map of chromosome 7A indicated that $Q f h s . f c u-7 A L$ is located in the proximal region of the long arm. This is only the second FHB QTL to be identified in a tetraploid source, and it may be useful to combine it with the QTL Qfhs.ndsu-3AS in order to develop durum wheat germ plasm and cultivars with higher levels of FHB resistance.
\end{abstract} the durum cv. Langdon (LDN) and a disomic LDN-T. turgidum sp.
Fusarium head blight (FHB), commonly known as scab, is recognized as one of the most destructive diseases of durum (Triticum turgidum L., $2 \mathrm{n}=4 \mathrm{x}=28$, AABB genomes) and common wheat ( $T$. aestivum L., $2 \mathrm{n}=6 \mathrm{x}=42$, AABBDD genomes) throughout the world, especially in areas with a warm and humid climate during anthesis. FHB infection of spikes causes sterile florets and shriveled kernels resulting in low yield, and the quality reduction in infected wheat grains is due to protein content change and contamination with several mycotoxins, most important of which is deoxynivalenol (DON) $(34,35)$. Changes in crop management practices including minimum or reduced tillage for stubble retention, weather (rainfall and high humidity periods) patterns, and low levels of resistance among current cultivars are considered the most important causes for severe FHB epidemics in the United States and Canada since $1993(8,19)$.

The deployment of FHB-resistant cultivars with good agronomic and quality characteristics is an effective, economical and environmentally sound way to reduce economic losses caused by FHB (19), but breeding efforts have been hindered by lack of effective resistance genes, the complex nature of inheritance for resistance in the identified resistant sources (21), confounding environmental effects, variation in pathogen species and aggressiveness, and laborious disease evaluation procedures. To date, most research on FHB resistance has concentrated on hexaploid common wheat for which resistant sources have been identified and are currently in use. The Chinese cv. Sumai 3 has been the

Corresponding author: J. D. Faris; E-mail address: farisj@ fargo.ars.usda.gov

\section{doi:10.1094/PHYTO-97-5-0592}

This article is in the public domain and not copyrightable. It may be freely reprinted with customary crediting of the source. The American Phytopathological Society, 2007 most extensively characterized hexaploid source of FHB resistance, and it has been used successfully as a source of resistance worldwide. A major quantitative trait locus (QTL) from Sumai 3 was identified on the wheat chromosome arm 3BS and designated Qfhs.ndsu-3BS (33), and the QTL was later validated by others $(1,2,5,39)$. This QTL, now considered a single gene designated $F h b l$, has since been the subject of extensive genomic analysis and fine mapping $(7,14,15)$.

Attempts to transfer resistance from Sumai 3 to durum wheat have met with limited success. However, one potential source of resistance for durum wheat is the tetraploid wild emmer wheat (T. turgidum sp. dicoccoides [Körn. ex Asch. \& Graebner] Aarons $[2 \mathrm{n}=4 \mathrm{x}=28$, AABB genomes]). It is a wild tetraploid wheat native to the Middle East and readily hybridizes with T. turgidum. Three T. turgidum sp. dicoccoides accessions (Israel A, PI478742, and PI481521) have been used to create disomic chromosome substitution lines in the 'Langdon' (LDN) durum background (11). The substitution lines provide valuable tools for evaluating the effects of individual chromosomes in isolation. The LDN-DIC Israel A (IsA) substitution lines were tested for reaction to FHB and the results indicated that the line having a pair of T. turgidum sp. dicoccoides $3 \mathrm{~A}$ chromosomes substituted for the pair of LDN $3 \mathrm{~A}$ chromosomes [LDN-DIC $3 \mathrm{~A}(\mathrm{IsA})$ ] was resistant with a mean FHB severity of $19.8 \%$ (32) compared with $50.8 \%$ for the moderately susceptible LDN. Otto et al. (25) used a mapping population of recombinant inbred (RI) chromosome lines constructed using LDN and LDN-DIC 3A(IsA) as parents to identify a QTL (Qfhs.ndsu-3AS) on chromosome arm 3AS that explained 37\% of the phenotypic variation for FHB resistance. Additional characterization and saturation mapping of this QTL led to the identification of markers explaining over $50 \%$ of the phenotypic variation (6). To date, Qfhs.ndsu-3AS is the only QTL identified in tetraploid wheat. 
Miller et al. (24) screened 290 accessions of T. turgidum sp. dicoccoides for reaction to FHB. Accessions PI481521 and PI478742 were found to have moderate levels of type II resistance (resistance to spread) and were used to construct disomic chromosome substitution lines in the LDN background (L. R. Joppa, unpublished data). Screening of the substitution lines for reaction to FHB in the greenhouse indicated that chromosome 7A, from both accessions, harbored resistance factors. Therefore, PI481521 and PI478742 seem to possess FHB resistance genes different from those identified in the T. turgidum sp. dicoccoides IsA accession. Here, our objectives were to develop a genetic linkage map of chromosome 7A in a population of RI lines (RILs) using simple sequence repeat (SSR) markers, and identify putative FHB resistance QTL on chromosome 7A derived from T. turgidum sp. dicoccoides PI478742.

\section{MATERIALS AND METHODS}

Plant material. The population consisted of 123 RILs derived from the cross between euploid LDN and LDN that had a pair of T. turgidum sp. dicoccoides PI478742 7A chromosomes substituted for the native 7A chromosomes [LDN-DIC 7A(742)]. Therefore, the RILs of this population contain a pair of recombinant 7A chromosomes in a homozygous LDN background. All RILs were derived from a single $F_{1}$ plant. The $F_{2}$ generation was advanced by the single seed decent method to the $\mathrm{F}_{7}$ generation, after which seed was bulked for each line. Of the 123 RILs, a subset of 118 RILs was used for molecular mapping and QTL analysis. In addition to the RI population and parents, the genotypes LDN-DIC 3A(IsA) (FHB-resistant), LDN-DIC 2A(IsA) (FHB-susceptible), and Ben (FHB-susceptible) were used as controls in the FHB inoculation experiments.

Preparation of Fusarium inoculum. During anthesis, plants were inoculated with a mixture of three isolates of $F$. graminearum (RWS R-1267, RWS R-1322, and R-010). The three isolate mixture was used to initially identify the resistance contributed by LDN-DIC 7A(742) and was therefore used for this study as well. Each isolate was originally isolated from symptomatic plants and was repeatedly tested for pathogenicity under a range of diverse environments. Each isolate was maintained, cultured, and prepared separately. The cultures of $F$. graminearum were grown on carnation leaf agar media (10). Conidia were produced on petri plates of culture media inoculated with different isolates of $F$. graminearum and then incubated at room temperature (22 to $25^{\circ} \mathrm{C}$ ) for 7 to 10 days. To prepare conidiospore inoculum, the conidia from the petri dishes were washed off with sterile distilled water. The conidial suspension then was strained through four layers of cheesecloth to remove mycelial fragments. The concentration was determined with a hemacytometer and adjusted to 50,000 conidia $\mathrm{ml}^{-1}$, and conidial suspensions of the three isolates were mixed together at equal concentrations immediately before use. Fresh conidial suspensions were held on ice and used within 2 to $3 \mathrm{~h}$.

FHB evaluations. FHB reactions of the 123 RILs along with the parents and checks were evaluated in the greenhouse under controlled conditions in three environments (fall 2004, spring 2005, and fall 2005) with four replicates per environment using a completely randomized design with checks within replicates. For fall 2004 there was one plant per pot, and for spring 2005 and fall 2005 there were two plants per pot with each pot considered an experimental unit. The parents and the checks were planted after every 20 RILs; therefore, each replication included six experimental units of parents and checks, which ensured that at least one resistant and susceptible check was inoculated on each day that RILs were inoculated.

As the plants reached anthesis, two or more spikes per line at the same stage of development in each replicate were inoculated by placing $10 \mu \mathrm{l}$ of conidial suspension within the glumes of a single flowering spikelet near the middle of each selected spike (33). This method of inoculation is selective for FHB type II resistance (resistance to spread). Following inoculation, a gentle overhead mist was applied for three consecutive nights. Three weeks after inoculation, spikes were scored individually for FHB symptoms on a scale of 0 to $100 \%$. The disease severity was represented as the percentage of necrotic spikelets. In all environments, artificial lighting was used to provide a $16-\mathrm{h}$ photoperiod. Temperature was maintained at approximately $25 \pm 2{ }^{\circ} \mathrm{C}$ in all environments.

Molecular mapping. A subset of 118 RILs was used for mapping and QTL analysis. DNA was extracted from RILs and parents using previously described methods (9). SSR primers developed by the U.S. Department of Agriculture-Agricultural Research Service, Beltsville Agricultural Research Center (BARC) (30), GWM SSR primer pairs mapped to chromosome 7A (27), and other available SSR primer pairs such as CFA (31) and KSUM (37) known to be mapped on chromosome 7A of wheat were screened for polymorphism among the parents of the RI population. Polymerase chain reaction (PCR) amplification of SSRs was done as previously described (27). PCR products were separated on $6 \%$ nondenaturing polyacrylamide gels, stained with SYBR Green II (Sigma Chemical, St. Louis), and visualized using a Typhoon 9410 variable mode imager (Molecular Dynamics Inc., Ithaca, NY). All the polymorphic SSR markers on chromosome 7A were used to genotype the RI population. The computer program MAPMAKER (13) version 2.0 for Macintosh was used to calculate linkage of the molecular markers using the Kosambi mapping function (12) and a minimum likelihood odds $(\mathrm{LOD})=3.0$ as described in Liu et al. (16).

Statistical analysis. The statistical analysis was done using SAS software version 9.1 (SAS Institute, Cary, NC). Data were analyzed by an unbalanced analysis of variance (ANOVA) using PROC GLM procedure of SAS. The phenotypic data from individual greenhouse environments as well as the combined data set across all three environments were evaluated.

For individual environment analysis, the data were analyzed as a completely randomized design using an entries-within-date arrangement. Therefore, the statistical model described genotypic effects only, and calculating differences among replications within each environment was not necessary. Combined ANOVA was done to test for the presence of significant experiment $\times$ genotype interactions. The statistical model for the data set combined across all three environments described the effects of genotype, environment, and genotype $\times$ environment interaction. Correlation coefficients for FHB severity means among the three environments were calculated using the Pearson product-moment procedure.

Mean separation for the genotypic means was determined by Fisher's protected least significant difference (LSD) at $\alpha=0.05$. Also, the parents and checks were analyzed as a separate data set to calculate the LSD for mean separation between checks and parents.

Segregation ratios of the markers were tested for fit to the expected 1:1 ratio by performing chi-square analysis on marker genotypes. The heritability of type II FHB resistance was calculated with the variance components from the combined analysis of FHB severity using the following formula:

$$
h^{2}=\frac{\sigma_{G}^{2}}{\sigma_{G}^{2}+\sigma_{G E}^{2} / e+\sigma_{\varepsilon}^{2} / r e}
$$

QTL analysis. RILs were scored as one of the two parental types for DNA markers. For each RIL, mean FHB severities were compared between the two DNA marker classes to identify significant differences. Linear and interval regression analysis was done using the computer program MapManager QTXb20 (17). Markers significant at the 0.001 level of probability were con- 
sidered to be significantly associated with resistance loci. Composite and simple interval regression analyses were done to identify marker intervals containing putative QTL associated with FHB resistance. To determine the critical LOD threshold, a permutation test with 1,000 permutations was executed. A LOD threshold of about 2.0 in this RIL population yielded an experiment-wise significance level of 0.05 . The coefficient of determination $\left(R^{2}\right)$ is the proportion of the total phenotypic variation explained by the markers. The mean FHB severity scores for each greenhouse environment as well as the means of combined FHB severity scores across the three environments were used.

\section{RESULTS AND DISCUSSION}

FHB evaluations. Significant differences in FHB severities were observed among the parents and checks, which all showed levels of infection that were in close agreement with previous experiments $(25,32)$. LDN is known to be only moderately susceptible to FHB and generally exhibits FHB severities of about $50 \%$, which agrees with the mean of the combined data in this study. Our preliminary experiments have consistently demonstrated that the LDN-DIC 7A(742) parent shows FHB severities of 25 to $30 \%$. In this study, LDN-DIC 7A(742) had a combined mean FHB severity of $26.9 \%$, which agrees with the preliminary work and indicates that the substitution of chromosome 7A from T. turgidum sp. dicoccoides accession PI478742 decreases FHB severity in LDN by about $50 \%$.

The frequency of the FHB severity in the RI population for individual environments (data not shown) as well as averaged over the three environments resembled a normal distribution (Fig. 1). In fall 2004 the mean FHB severity for the RI population was $64.4 \%$, and FHB severities ranged from 12 to $100 \%$ (Table 1). Population means for FHB severity during spring 2005 and fall 2005 were 36.0 and $40.2 \%$, respectively, and the population ranges of FHB severity for the same two environments were 15.4 to 72.0 and 26.6 to 61.9 , respectively. The mean FHB severity for the three environments combined was $46.9 \%$ with a range of 20.2 to $75.6 \%$. Analysis of variance for the three environments combined indicated there were no significant genotype $\times$ environment interactions (Table 2).

Lines with significantly greater resistance than that of LDNDIC 7A did not exist, but a few transgressive susceptible segregants were found (Fig. 1), which were a result of these lines

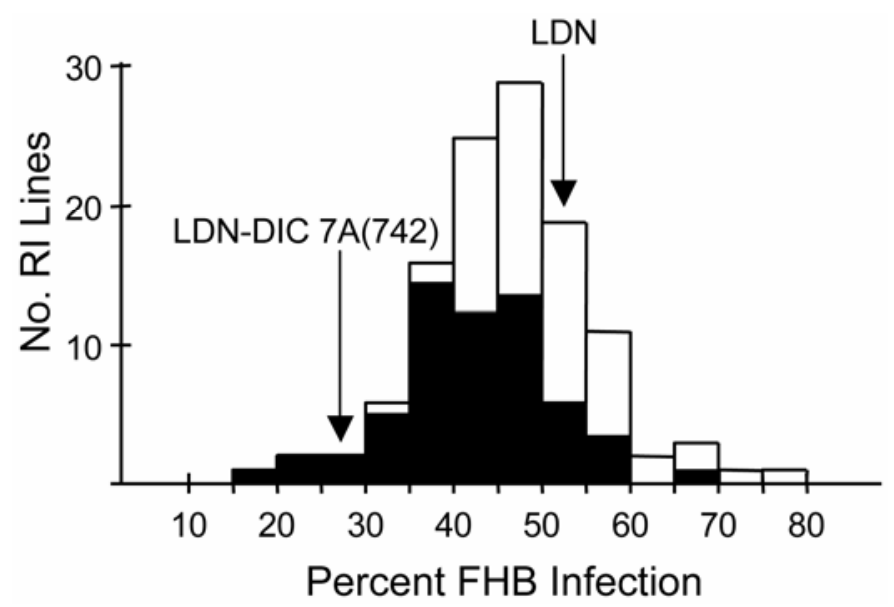

Fig. 1. Histogram of Fusarium head blight severities in the recombinant inbred (RI) population derived from cv. Langdon $(\mathrm{LDN}) \times \mathrm{LDN}-\mathrm{DIC} 7 \mathrm{~A}(742)$. These data represent average severities combined from the three greenhouse seasons of evaluation. White bars represent the frequency of the whole population, and black bars represent RI lines carrying the LDN-DIC 7A(742) allele at the Xbarc121 locus. showing highly susceptible reactions in fall 2004, but more moderately susceptible reactions in spring 2005 and fall 2005. Analysis of correlations of FHB severity means among the three environments indicated that the fall 2004 environment did not correlate well with the spring and fall 2005 environments (Table $3)$. The narrow-sense heritability $\left(h^{2}\right)$ for the LDN-DIC 7A(742) RI population for type II FHB resistance was 0.2 for fall 2004 and spring 2005 environments, 0.4 for the fall 2005 environment, and 0.3 for the three environments combined.

Genetic linkage mapping of chromosome 7A. A total of 15 out of $48(31 \%)$ SSR primer pairs surveyed on the parents of the RI population were polymorphic. The 15 primer sets detected 16 marker loci, which were assembled into a linkage map that spanned a genetic distance of $172.9 \mathrm{cM}$ (Fig. 2). The average marker density of this map was one marker per $10.8 \mathrm{cM}$. Three markers (Xgwm130, XksuM176, and Xgwm282) showed significant deviation from the expected Mendelian segregation ratio of $1: 1$ at the 0.05 level of probability. The largest gap on the map was $31.2 \mathrm{cM}$ between markers Xksum176 and Xbarc121 on the long arm of the chromosome. The position of the centromere was determined using the information available from previously published SSR maps in wheat and was established to lie between markers Xbarc1034 and Xbarc108 (16,27,31).

The linkage map of chromosome 7A was compared with the physical map of chromosome 7A based on chromosome deletion breakpoints (31) (Fig. 2). Eleven of the sixteen SSR marker loci used to construct the 7A genetic linkage map were also placed on the 7A physical map. The order of common markers along the genetic map was in complete agreement with that of the physical map with one exception. Marker Xcfa2257 detected a locus at the

TABLE 1. Mean Fusarium head blight severity (percent spread or type II reaction) for parents, 123 recombinant inbred lines, and checks evaluated in the greenhouse at Fargo, ND, in the fall 2004, spring 2005, and fall 2005

\begin{tabular}{lllll}
\hline & Fall 2004 & Spring 2005 & Fall 2005 & Combined \\
\hline LDN & 66.3 & 38.5 & 46.0 & 50.3 \\
LDN DIC 7A & 36.5 & 19.2 & 24.8 & 26.9 \\
LDN DIC 3A & 12.3 & 9.1 & 7.9 & 9.8 \\
LDN DIC 2A & 94.9 & 83.9 & 92.5 & 90.4 \\
BEN & 93.8 & 70.4 & 88.6 & 84.3 \\
Population mean & $64.4 \pm 16.6$ & $36.0 \pm 11.0$ & $40.2 \pm 7.3$ & $46.9 \pm 8.5$ \\
Population range & $12.2-100.0$ & $15.4-72.0$ & $26.5-61.8$ & $20.2-75.6$ \\
Coefficient & & & & \\
$\quad$ of variation & 34.4 & 34.8 & 12.4 & 32.9 \\
LSD & 12.0 & 8.8 & 3.7 & 5.6 \\
\hline
\end{tabular}

a The least significant difference (LSD) refers to differences among means of parents and checks.

TABLE 2. Analysis of variance for Fusarium head blight severity of the cv. Langdon $(\mathrm{LDN}) \times \mathrm{LDN}$-DIC $7 \mathrm{~A}(742)$ recombinant inbred population for the fall 2004, spring 2005, and fall 2005 environments combined ${ }^{\mathrm{a}}$

\begin{tabular}{lrccc}
\hline Source & \multicolumn{1}{c}{ df } & Mean square & $F$ value & $P$ value \\
\hline Experiment & 2 & $92,693.6$ & 175.2 & $<0.0001$ \\
Genotype & 122 & $3,787.2$ & 7.2 & $<0.0001$ \\
Experiment $\times$ genotype & 244 & 527.3 & 1.0 & 0.5046 \\
Error & 1,340 & 529.1 & $\ldots$ & $\ldots$ \\
Corrected total & 1,708 & $\ldots$ & $\ldots$ & $\ldots$ \\
\hline
\end{tabular}

${ }^{\text {a }}$ Coefficient of variation $=48.6$.

TABLE 3. Correlation coefficients for type II (resistance to spread) Fusarium head blight severity means among the fall 2004, spring 2005, and fall 2005 environments

\begin{tabular}{lcc}
\hline Environment & Fall 2004 & Spring 2005 \\
\hline Fall 2004 & $\ldots$ & $\ldots$ \\
Spring 2005 & 0.10 & $\ldots$ \\
Fall 2005 & 0.26 & 0.78 \\
\hline
\end{tabular}


distal end of our chromosome 7A linkage group, but it mapped at a more proximal location on the physical map.

QTL analysis. In single marker regression analysis using phenotypic means combined across all environments, Xbarc121 was the marker most significantly $(P<0.00001)$ associated with the FHB resistance, and it explained $17 \%$ of the phenotypic variation. The markers Xbarc49, Xbarc29.1, and Xbarc29.2 were also significantly $(P<0.001)$ associated with FHB resistance using combined phenotypic means and explained 10 to $12 \%$ of the phenotypic variation. Single marker regression analysis using phenotypic means from individual environments indicated that Xbarc121 was significantly $(P<0.001)$ associated with FHB resistance in the fall 2004 and spring 2005 environments and explained 10 and $12 \%$ of phenotypic variation, respectively. No markers were significantly associated with FHB phenotypic means obtained from the fall 2005 environments at the 0.001 level of probability, but marker Xbarc121 had a $P$ value of 0.019 and explained $5 \%$ of the variation.

Interval regression analysis using combined phenotypic means indicated that Xbarc121 defined the peak of the QTL located on chromosome 7A (Fig. 2). We propose to designate this QTL as Qfhs.fcu-7AL according to McIntosh et al. (18). Qfhs.fcu-7AL spanned an interval of $39.6 \mathrm{cM}$ and explained $19 \%$ of the phenotypic variation for FHB resistance with a LOD score of 5.2. The same genomic region was identified using interval regression mapping for the fall 2004 and spring 2005 environments (Fig. 2). For the fall 2004 data, Qfhs.fcu-7AL peaked between SSR markers Xksum 176 and Xbarc121 with a LOD $\geq 2$, and explained $12 \%$ of the phenotypic variation. For the spring 2005 data, Qfhs.fcu-7AL peaked near marker Xbarc121 at LOD $\geq 2$ and explained $14 \%$ of the phenotypic variation. No significant QTL was detected using the fall 2005 data, but there was some association found at LOD $<2$ for the marker Xbarc121, which explained $6 \%$ of the phenotypic variation.

The RI population used in the identification of $Q f h s . f c u-7 A L$ segregated for only chromosome 7A. In studies conducted on such a population structure it is expected to find the resistance factors explaining much of the variation conferred by the resistant parent. Qfhs.fcu-7AL explained only $19 \%$ of the phenotypic variation and was not significant in fall 2005 using the thresholds

\section{A Physical Map}

\section{Deletion breakpoints}

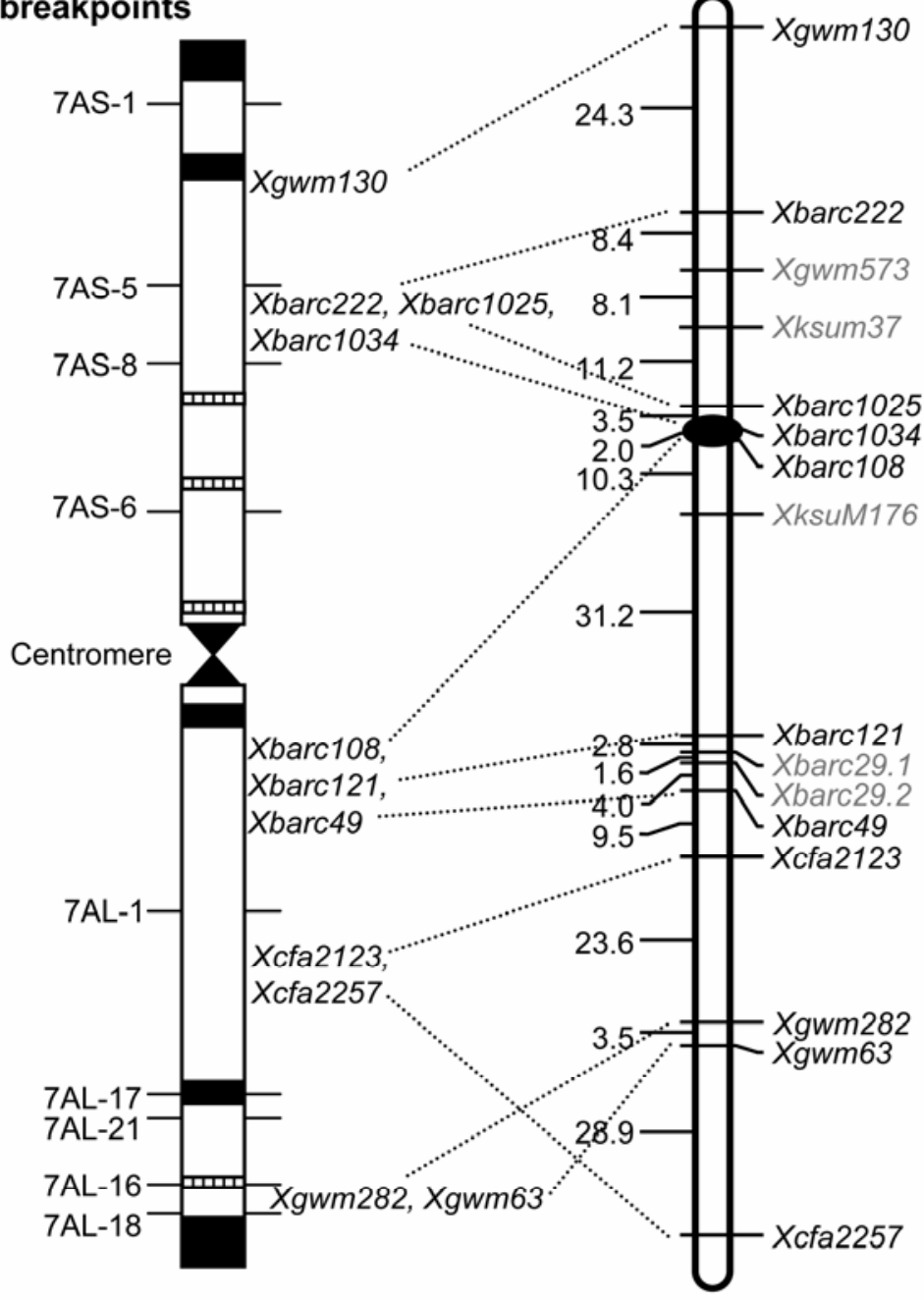

\section{A Genetic Map}

\section{A Interval Regression Map}

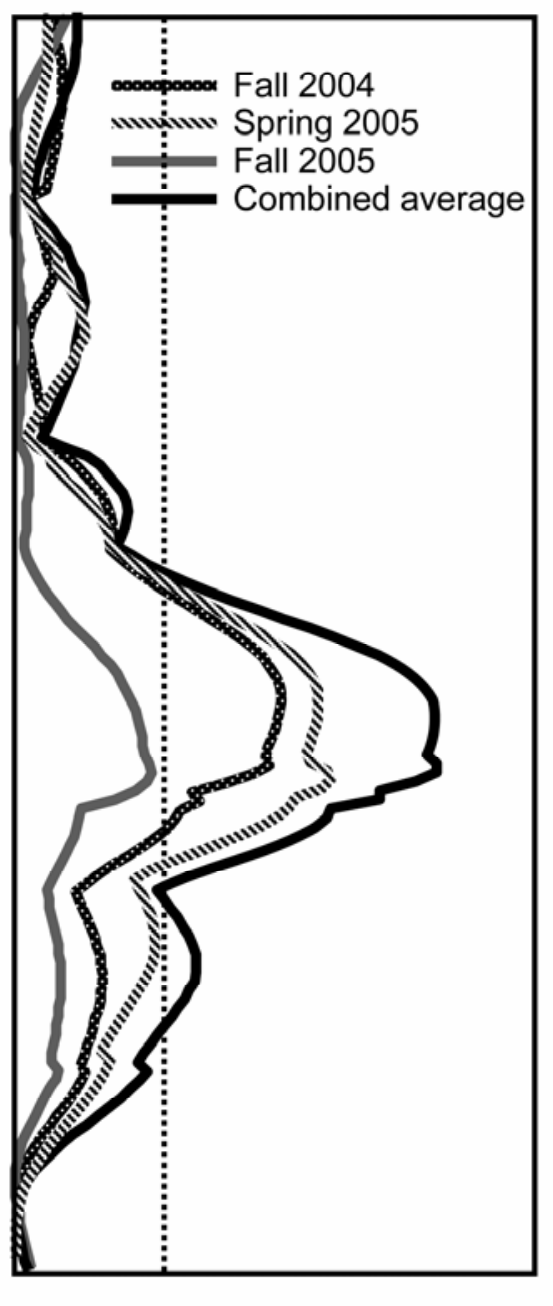

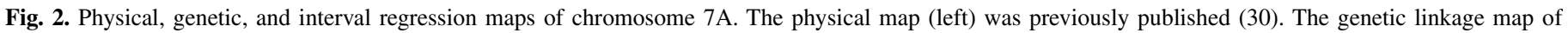

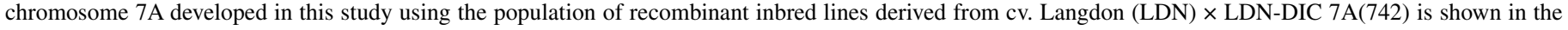

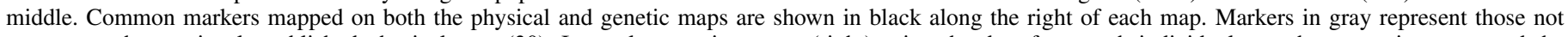

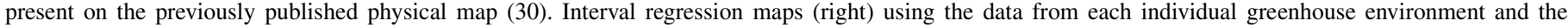

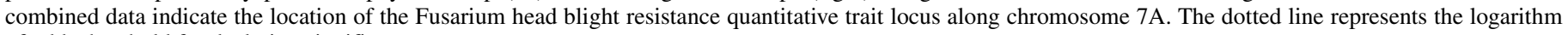
of odds threshold for declaring significance. 
chosen for this study. The reason behind this observation is likely due to the inherent complexities of conducting and evaluating controlled FHB inoculations and likely reflects environmental effects including interactions with genotypes, pathogen isolates, inoculation methods, and methods of scoring disease phenotypes. Nevertheless, a QTL explaining 19\% of the variation for FHB resistance is quite large relative to many FHB QTL, and evaluations of more replications and the identification of more closely linked markers might lead to $Q f h s . f c u-7 A L$ explaining a greater portion of the phenotypic variation.

Composite interval mapping indicated that only one QTL exists on chromosome 7A in this population. However, it is possible that additional minor factors affecting FHB resistance reside on chromosome 7A but were undetected due to the relatively small population size.

Comparisons of the markers associated with the QTL on the genetic map with the physical map of chromosome 7A (31) indicated that the QTL identified in this study lies on the long arm of chromosome 7A near the centromere. The markers showing significant association with the FHB resistance are located between the centromere and deletion breakpoint $7 \mathrm{AL}-1$ on the physical map (Fig. 2).

Various studies have identified genomic factors responsible for resistance to FHB on chromosome 7A or homologous group 7 $(4,36,38)$ using various genetic and cytogenetic tools. More recently, Zhou et al. (39) found that chromosome 7A from Sumai 3 showed the largest effect on resistance to FHB spread and DON accumulation in Chinese Spring (Sumai 3) chromosome substitution lines. Zhou et al. (40) identified a minor QTL on 7AL that explained $9.8 \%$ of the phenotypic variation. The QTL was flanked by the SSR marker Xwms1083 and the amplified fragment length polymorphism (AFLP) marker $p C G A / m T G C G 231$. Until now, no extensive study to identify QTL on chromosome 7A of tetraploid

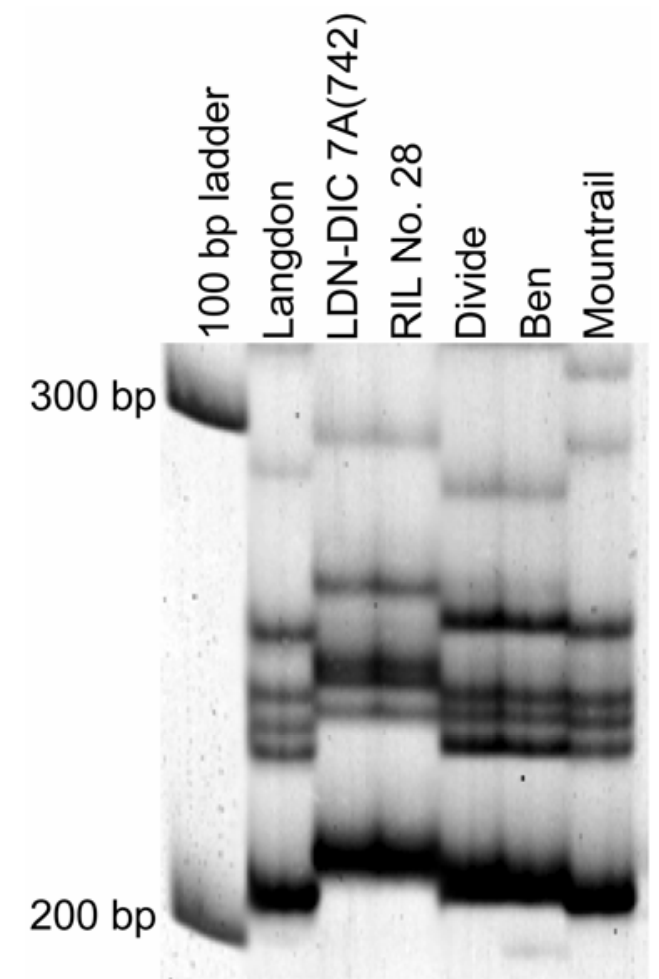

Fig. 3. Polyacrylamide gel electrophoresis of polymerase chain reactionamplified fragments corresponding to the marker Xbarc121. The genotypes including Langdon, Langdon-Triticum turgidum sp. dicoccoides PI478742 disomic chromosome 7A substitution line [LDN-DIC 7A(742)], the most resistant recombinant inbred line (RIL 28), and the durum cvs. Divide, Ben, and Mountrail, are annotated across the top, and the 200 and $300 \mathrm{bp}$ size markers are noted on the left. wheat has been conducted. The QTL Qfhs. $f c u-7 A L$ showed its highest peak at Xbarc121 and is located in deletion bin C-7AL $1 \sim 0.39$. Due to the limitation of available physical maps in wheat, the physical location of SSR marker Xwms 1083 and AFLP marker $p C G A / m T G C G 231$ identified by Zhou et al. (41) could not be established to compare the identity of $Q f h s . f c u-7 A L$ with the FHB-resistant QTL identified by Zhou et al. (40).

Shen et al. (28) evaluated type II FHB resistance in 19 Chinese Spring-Lophopyrum elongatum (EE) substitution lines, two Thatcher-L. ponticum $\left(7 \mathrm{el}_{1}\right.$ and $\left.7 \mathrm{el}_{2}\right)$ substitution lines, and four Thatcher- $L$. ponticum translocation lines. Substitution lines $7 \mathrm{E}$ (7A), 7E (7B), and 7E (7D) consistently had the lowest disease severity across the three experiments. The homologous chromosome $7 \mathrm{el}_{2}$ also showed resistance in the Thatcher genetic background. In most plants containing $7 \mathrm{E}$, only the inoculated floret showed symptoms of scab as a small lesion.

The marker density on chromosome 7A in the present study was not sufficient to define the FHB resistance QTL into a small genomic region. The marker Xbarc 121, which detected a 210-bp fragment in LDN (Fig. 3), may be of use for marker-assisted selection, but more precise localization through marker enrichment of the Qfhs.fcu-7AL region would lead to increased efficiency of marker-assisted selection. In addition, the identification of more markers tightly linked to $Q f h s . f c u-7 A L$ would provide breeders and geneticists with more options for genotyping and selection. The physical location of $Q f h s . f c u-7 A L$ in deletion bin C-7AL 1 0.39 would be helpful for selecting additional markers potentially mapping within the QTL region. The available wheat physical maps, which include a large number of the SSR, restriction fragment length polymorphism, and expressed sequence tags mapped in the same deletion bin as $Q f h s . f c u-7 A L$, provide a rich source of markers to be used in future studies.

\section{ACKNOWLEDGMENTS}

We thank E. Doehler, J. Hanson, and P. Meyer for technical assistance, and R. Horsley and S. Xu for assistance with statistical analysis. This research was supported by the U.S. Wheat and Barley Scab Initiative via the U.S. Department of Agriculture-Agricultural Research Service (USDA-ARS) and USDA-ARS Current Research Information System project 5442-22000-030-00D.

\section{LITERATURE CITED}

1. Anderson, J. A., Stack, R. W., Liu, S., Waldron, B. L., Fjeld, A. D., Coyne, C., Moreno-Sevilla, B., Fetch, J. M., Song, Q. J., Cregan, P. B., and Frohberg, R. C. 2001. DNA markers for Fusarium head blight resistance QTLs in two wheat populations. Theor. Appl. Genet. 102:11641168.

2. Bai, G.-H., Kolb, F. L., Shaner, G., and Domier, L. L. 1999. Amplified fragment length polymorphism markers linked to a major quantitative trait locus controlling scab resistance in wheat. Phytopathology 89:343-348.

3. Bai, G.-H., and Shaner, G. 2004. Management and resistance in wheat and barley to Fusarium head blight. Annu. Rev. Phytopathol. 42:135-161.

4. Buerstmayr, H., Lemmens, M., Fedak, G., and Ruckenbauer, P. 1999. Back-cross reciprocal monosomic analysis of Fusarium head blight resistance in wheat (Triticum aestivum L.). Theor. Appl. Genet. 98:76-85.

5. Buerstmayr, H., Lemmens, M., Hartl, L., Doldi, L., Steiner, B., Stierschneider, M., and Ruckenbauer, P. 2002. Molecular mapping of QTLs for Fusarium head blight resistance in spring wheat. I. Resistance to fungal spread (Type II resistance).Theor. Appl. Genet. 104:84-91.

6. Chen, X. F., Faris, J. D., Hu, J. G., Stack, R. W., Adhikari, T., Elias, E. M., Kianian, S. F., and Cai, X. W. 2006. Saturation and comparative mapping of a major Fusarium head blight resistance QTL in tetraploid wheat. Mol. Breed. 19:113-124.

7. Cuthbert, P. A., Somers, D. J., Thomas, J., Cloutier, S., and Brule-Babel, A. 2006. Fine mapping Fhb1, a major gene controlling Fusarium head blight resistance in bread wheat (Triticum aestivum L.). Theor. Appl. Genet. 112:1465-1472.

8. Dill-Macky, R., and Jones, R. K. 1997. The effect of previous crops and tillage on Fusarium head blight of wheat. Cereal Res. Comm. 25:711-712.

9. Faris, J. D., Haen, K. M., and Gill, B. S. 2000. Saturation mapping of gene-rich recombination hot spot region in wheat. Genetics 154:823-835. 
10. Fisher, N. L., Burgess, L. W., Toussoun, T. A., and Nelson, P. E. 1982. Carnation leaves as a substrate and for preserving cultures of Fusarium species. Phytopathology 72:151-153.

11. Joppa, L. R., and Cantrell, R. G. 1990. Chromosomal location of genes for grain protein content of wild tetraploid wheat. Crop Sci. 30:1059-1064.

12. Kosambi, D. D. 1944. The estimation of map distances from recombination values. Ann. Eugen. 12:172-175.

13. Lander, E. S., Green, P., Abrahamson, J., Barlow, A., Daly, M. J., Lincoln, S. E., and Newburg, L. 1987. Mapmaker: An interactive computer package for constructing primary genetic linkage maps of experimental and natural populations. Genomics 1:174-181.

14. Liu, S., and Anderson, J. A. 2003. Targeted molecular mapping of a major wheat QTL for Fusarium head blight resistance using wheat ESTs and synteny with rice. Genome 46:817-823.

15. Liu, S., Zhang, X., Pumphrey, M. O., Stack, R. W., Gill, B. S., and Anderson, J. A. 2006. Complex microcolinearity among wheat, rice, and barley revealed by the fine mapping of a genomic region harboring a major QTL for resistance to Fusarium head blight in wheat. Funct. Integr. Genomics 6:83-89.

16. Liu, Z., Anderson, J. A., Hu, J., Friesen, T. L., Rasmussen, J. B., and Faris, J. D. 2005. A wheat intervarietal linkage map based on microsatellite and target region amplified polymorphism markers and its utility for detecting quantitative trait loci. Theor. Appl. Genet. 111:782-794.

17. Manly, K. F., Cudmore, R. H., Jr., and Meer, J. M. 2001. Map manager QTX, cross-platform software for genetic mapping. Mamm. Genome 12:930-932.

18. McIntosh, R. A., Hart, G. E., Devos, K. M., Gale, M. D., and Rogers, W. J. 1998. Catalogue of gene symbols for wheat. In: International Wheat Genetics Symposium, 9th, Saskatoon. A. E. Slinkard, ed. University Extension Press, University of Saskatchewan, Saskatoon.

19. McMullen, M. P., Jones, R., and Gallenberg, D. 1997. Scab of wheat and barley: A re-emerging disease of devastating impact. Plant Dis. 81:13401348 .

20. Mesterhazy, A. 1995. Types and components of resistance to Fusarium head blight of wheat. Plant Breed. 114:377-386.

21. Mesterházy, Á. 1997. Methodology of resistance testing and breeding against Fusarium head blight in wheat and results of selection. Cereal Res. Commun. 25:631-637.

22. Mesterházy, Á. 2003. Breeding wheat for Fusarium head blight resistance in Europe. Pages 211-240 in: Fusarium Head Blight of Wheat and Barley. K. J. Leonard and W. R. Bushnell, eds. The American Phytopathological Society, St. Paul, MN.

23. Miedaner, T. 1997. Breeding wheat and rye for resistance to Fusarium diseases. Plant Breed. 116:201-220.

24. Miller, J. D., Stack, R. W., and Joppa, L. R. 1998. Evaluation of Triticum turgidum L. var. dicoccoides for resistance to Fusarium head blight and stem rust. Pages 292-293 in: Proc. IX Int. Wheat Genetics Symp., vol. 3., Saskatoon. A. E. Slinkard, ed. University of Saskatoon Extension Press, Saskatoon.

25. Otto, C. D., Kianian, S. F., Elias, E. M., Stack, R. W., and Joppa, L. R. 2002. Genetic dissection of a major Fusarium head blight QTL in tetraploid wheat. Plant Mol. Biol. 48:625-632.

26. Parry, D. W., Jenkinson, P., and McLeod, L. 1995. Fusarium ear blight (scab) in small grain cereals-A review. Plant Pathol. 44:207-238.

27. Röder, M. S., Korzun, V., Wendehake, K., Plaschke, J., Tixier, M. H., Leroy, P., and Ganal, M. W. 1998. A microsatellite map of wheat. Genetics 149:2007-2023.

28. Shen, X., Kong, L., and Ohm, H. 2004. Fusarium head blight resistance in hexaploid wheat (Triticum aestivum)-Lophopyrum genetic lines and tagging of the alien chromatin by PCR markers. Theor. Appl. Genet. 108:808-813.

29. Snijders, C. H. A. 1990. Genetic variation for resistance to Fusarium head blight in bread wheat. Euphytica 50:171-179.

30. Song, Q. J., Shi, J. R., Singh, S., Fickus, E. W., Costa, J. M., Lewis, J., Gill, B. S., Ward, R., and Cregan, P. B. 2005. Development and mapping of microsatellite (SSR) markers in wheat. Theor. Appl. Genet. 110:550560 .

31. Sourdille, P., Singh, S., Cadalen, T., Brown-Guedira, G. L., Gay, G., Qi, L., Gill, B. S., Dufour, P., Murigneux, A., and Bernard, M. 2004. Microsatellite-based deletion bin system for the establishment of geneticphysical map relationships in wheat (Triticum aestivum L.). Funct. Integr. Genomics 4:12-25.

32. Stack, R. W., Elias, E. M., Mitchell Fetch, J., Miller, J. D., and Joppa, L. R. 2002 Fusarium head blight reaction of Langdon durum-Triticum dicoccoides chromosome substitution lines. Crop Sci. 42:637-642.

33. Waldron, B. L., Moreno-Sevilla, B., Anderson, J. A., Stack, R. W., and Frohberg, R. C. 1999. RFLP mapping of QTL for Fusarium head blight resistance in wheat. Crop Sci. 39:805-811.

34. Wong, L. S. L., Abramson, D., Tekauz, A., Leisle, D., and Mckenzie, R. I. H. 1995. Pathogenicity and mycotoxin production of Fusarium species causing head blight in wheat cultivars varying in resistance. Can. J. Plant Sci. 75:261-267.

35. Wu, Y. L., Wu, G. Q., and Ye, H. Z. 1997. Changes in protein components of wheat grain infected with F. graminearum. J. Sichuan Agric. Univ. 15:329-334.

36. Yao, J., Ge, Y., Wang, S., Yao, G., Zhou, C., and Qian, C. 1997. Chromosomal location of genes for scab resistance in wheat cultivar Sumai 3. Acta Agron. Sin. 23:450-453.

37. Yu, J.-K., Dake, T. M., Singh, S., Benscher, D., Li, W., Gill, B. S., and Sorrells, M. E. 2004. Development and mapping of EST-derived simple sequence repeat markers for hexaploid wheat. Genome 47:805-818.

38. Yu, Y. J. 1991. Genetic analysis of scab resistance in five wheat varieties PHJZM, HHDTB, CYHM, YGFZ, and WN2. Acta Agron. Sin. 17:248253.

39. Zhou, W. C., Kolb, F. L., Bai, G.-H., Domier, L. L., and Yao, J. B. 2002. Effect of individual Sumai 3 chromosomes on resistance to scab spread within spikes and deoxynivalenol accumulation within kernels in wheat. Hereditas 137:81-89.

40. Zhou, W. C., Kolb, F. L., Yu, J., Bai, G., Boze, L. K., and Domier, L. L. 2004. Molecular characterization of Fusarium head blight resistance in Wangshuibai with simple sequence repeat and amplified fragment length polymorphism markers. Genome 47:1137-1143. 